\title{
$\beta$-Bursts Reveal the Trial-to-Trial Dynamics of Movement Initiation and Cancellation
}

\author{
기 Jan R. Wessel ${ }^{1,2}$ \\ ${ }^{1}$ Department of Psychological and Brain Sciences, University of Iowa, Iowa City, Iowa 52245, and ${ }^{2}$ Department of Neurology, University of Iowa Hospital \\ and Clinics, Iowa City, Iowa 52242
}

The neurophysiological basis of motor control is of substantial interest to basic researchers and clinicians alike. Motor processes are accompanied by prominent field potential changes in the $\beta$-frequency band (15-29 Hz): in trial-averages, movement initiation is accompanied by $\beta$-band desynchronization over sensorimotor areas, whereas movement cancellation is accompanied by $\beta$-power increases over (pre)frontal areas. However, averaging misrepresents the true nature of the $\beta$-signal. Unaveraged $\beta$-band activity is characterized by short-lasting, burst-like events, rather than by steady modulations. Therefore, averaging-based quantifications may miss important brain-behavior relationships. To investigate how $\beta$-bursts relate to movement in male and female humans $(N=234)$, we investigated scalp-recorded $\beta$-band activity during the stop-signal task, which operationalizes both movement initiation and cancellation. Both processes were indexed by systematic spatiotemporal changes in $\beta$-burst rates. Before movement initiation, $\beta$-bursting was prominent at bilateral sensorimotor sites. These burst-rates predicted reaction time (a relationship that was absent in trial-average data), suggesting that sensorimotor $\beta$-bursting signifies an inhibited motor system, which has to be overcome to initiate movements. Indeed, during movement initiation, sensorimotor burst-rates steadily decreased, lateralizing just before movement execution. In contrast, successful movement cancellation was signified by increased phasic $\beta$-bursting over fronto-central sites. Such $\beta$-bursts were followed by shortlatency increases of bilateral sensorimotor $\beta$-burst rates, suggesting that motor inhibition can be rapidly re-instantiated by frontal areas when movements have to be rapidly cancelled. Together, these findings suggest that $\beta$-bursting is a fundamental signature of the motor system, used by both sensorimotor and frontal areas involved in the trial-by-trial control of behavior.

Key words: $\beta$-bursts; EEG; motor inhibition; movement; response inhibition; stop-signal task

\section{Significance Statement}

Movement-related $\beta$-frequency $(15-29 \mathrm{~Hz}$ ) changes are among the most prominent features of neural recordings across species, scales, and methods. However, standard averaging-based methods obscure the true dynamics of $\beta$-band activity, which is dominated by short-lived, burst-like events. Here, we demonstrate that both movement-initiation and cancellation in humans are characterized by unique trial-to-trial patterns of $\beta$-bursting. Movement initiation is characterized by steady reductions of $\beta$-bursting over bilateral sensorimotor sites. In contrast, during rapid movement cancellation, $\beta$-bursts first emerge over frontocentral sites typically associated with motor control, after which sensorimotor $\beta$-bursting re-initiates. These findings suggest a fundamentally novel, non-invasive measure of the neural interaction underlying movement-initiation and -cancellation, opening new avenues for the study of motor control in health and disease.

\section{Introduction}

Activity in the $\beta$-frequency band $(15-29 \mathrm{~Hz})$ is a prominent constituent of the neural local field potential. It can be observed at

\footnotetext{
Received Aug. 3, 2019; revised Nov. 11, 2019; accepted Nov. 12, 2019.

Author contributions: J.R.W. designed research; J.R.W. performed research; J.R.W. analyzed data; J.R.W. wrote the paper.

This research was supported by Grants from the National Institute of Health (R01 NS102201), the National Science Foundation (CAREER 1752355), and the Roy J. Carver Charitable Trust (Research Program of Excellence 17-4885). I thank Daniel Thayer, Julian Scheffer, Cailey Parker, Hailey Billings, Carly Ryder, Megan Hynd, Carly lacullo, Brynne Dochterman, Isabella Dutra, Alec Mather, Kylie Dolan, Nathan Chalkley, Darcy Waller, Tobin Dykstra, and Cheol Soh for help with data collection; Mark Blumberg, John Freeman, and Bob McMurray for helpful discus-
}

spatial scales ranging from extracellular to scalp recordings, in species ranging from rodents to humans, and using methods ranging from intracranial recordings to magnetoencephalography (Murthy and Fetz, 1992; Sanes and Donoghue, 1993; Engel and Fries, 2010; Shin et al., 2017). The $\beta$-frequency plays a par-

\footnotetext{
sions; and Freek van Ede, Eric Maris, and Jan-Mathijs Schoffelen for help with the lagged coherence method, and Bernhard Spitzer for suggesting it.

The author declares no competing financial interests.

Correspondence should be addressed to Jan R. Wessel at Jan-wessel@uiowa.edu.

https://doi.org/10.1523/JNEUROSCI.1887-19.2019

Copyright $\odot 2020$ the authors
} 
ticularly important role in motor functions. In particular, during movement initiation, a prominent desynchronization of $\beta$-band activity is clearly observable over sensorimotor areas (McFarland et al., 2000; Pfurtscheller et al., 2003). In contrast, the rapid cancellation of movements is accompanied by $\beta$-power increases over (pre)frontal cortical areas typically implicated in cognitive control (Swann et al., 2009, 2011, 2012; Picazio et al., 2014; Wagner et al., 2018). Moreover, movement-related changes in $\beta$-power can also be observed in extrapyramidal parts of the motor system, including the basal ganglia (Kühn et al., 2004; Ray et al., 2012; Brittain and Brown, 2014; Wessel et al., 2016), where abnormal $\beta$-rhythms are prominently observed in movement disorders such as Parkinson's disease (Hammond et al., 2007; Bronte-Stewart et al., 2009; Jenkinson and Brown, 2011; Little and Brown, 2014; Quinn et al., 2015).

Recent studies of raw, unaveraged $\beta$-band activity, however, have led to a significant reappraisal of the nature of neural activity in the $\beta$-band. Whereas trial-averaging approaches have long suggested that movement-related changes in the $\beta$-band are expressed in steady (de)synchronizations that stretch over several hundred milliseconds, un-averaged $\beta$-band activity is primarily characterized by transient burst-like events, which typically last $<\sim 150$ ms (Leventhal et al., 2012; Feingold et al., 2015; Sherman et al., 2016). Although these burst-events look like slow-evolving (de)synchronizations when averaged across trials, analyses of single-trial data have found that the simple presence or absence of these $\beta$-bursts, rather than overall changes in $\beta$-power, is the most reliable predictor of trial-to-trial behavior (Shin et al., 2017).

Here, we therefore investigated the characteristics of singletrial $\beta$-bursting in humans during both the initiation and the rapid cancellation of movement. A large sample of healthy human participants $(N=234)$ performed the stop-signal task (Logan and Cowan, 1984; Verbruggen et al., 2019), a motor task that includes both instances of movement initiation (following a gosignal) and movement cancellation (on a subset of trials that include a subsequent stop-signal). We used non-invasive scalpEEG recordings to investigate how $\beta$-bursting on individual trials indexes both processes, as well as their interaction. Specifically, we investigated four questions: (1) Is human cortical $\beta$-band activity during movement burst-like? (2) If so, do systematic spatiotemporal patterns of $\beta$-burst activity distinguish successful from unsuccessful movement cancellation, as would be expected based on the trial-average literature on $\beta$-power? (3) Are there systematic relationships between initiation and cancellationrelated changes in $\beta$-bursting when movements have to be rapidly stopped? (4) Do $\beta$-bursts provide a more accurate representation of brain-behavior relationships compared with changes in overall $\beta$-amplitude?

\section{Materials and Methods}

\section{Participants}

Two hundred and thirty-four healthy adult humans (mean age: 22.7, SEM: 0.43, 137 female, 25 left-handed) from the Iowa City community participated in the study, either for course credit or for an hourly payment. The majority of these datasets have been previously published as part of other studies, none of which focused on $\beta$-bursting (Dutra et al., 2018; Wessel, 2018a,b; Dykstra et al., 2019; Waller et al., 2019; Wessel et al., 2019). All procedures were approved by the local ethics committee at the University of Iowa (IRB 201511709).

Task

The task was identical to the one described by Wessel, (2018a,b), Dutra et al. (2018), and Waller et al. (2019). In short, trials began with a fixation cross (500 ms duration), followed by a white leftward or rightward arrow (Go-signal). Participants were instructed to respond as fast and accurately as possible to the arrow using their left or right index finger (the respective response buttons were $\mathrm{q}$ and $\mathrm{p}$ on a QWERTY keyboard). On one-third of trials, a Stop-signal occurred (the arrow turned from white to red) at a delay after the go-stimulus [stop-signal delay (SSD)]. The SSD, which was initially set to $200 \mathrm{~ms}$, was dynamically adjusted in $50 \mathrm{~ms}$ increments to achieve a p(stop) of 0.5: after successful stops, the SSD was prolonged; after failed stops, it was shortened. This was done independently for leftward and rightward go-stimuli. Trial duration was fixed at $3000 \mathrm{~ms}$. Six blocks of 50 trials were performed (200 Go, 100 Stop).

\section{Data availability}

All data, procedures, and analysis routines can be downloaded on the Open Science Framework at https://osf.io/v3a78/.

\section{EEG recording}

Scalp-EEG was recorded using two different Brain Products systems; one active (actiChamp) and one passive (MR plus). In both cases, 62-channel electrode caps with two additional electrodes on the left canthus (over the lateral part of the orbital bone of the left eye) and over the part of the orbital bone directly below the left eye were used. The ground was placed at electrode $\mathrm{Fz}$, and the reference was placed at electrode Pz. EEG was digitized at a sampling rate of $500 \mathrm{~Hz}$, with hardware filters set to $10 \mathrm{~s}$ time-constant high-pass and $1000 \mathrm{~Hz}$ low-pass.

\section{EEG data preprocessing}

Data were preprocessed using custom routines in MATLAB, incorporating functions from the EEGLAB toolbox (Delorme and Makeig, 2004; RRID:SCR_007292). The electrode $\times$ time-series matrices for each task were imported into MATLAB and then filtered using symmetric two-way least-squares finite impulse response filters (high-pass cutoff: $0.3 \mathrm{~Hz}$, low-pass cutoff: $30 \mathrm{~Hz}$ ). Non-stereotyped artifacts were automatically removed from further analysis using segment statistics applied to each second-long segment of data (joint probability and joint kurtosis, with both cutoffs set to 5 SD, cf. Delorme et al., 2007). Trials that included a rejected data segment were excluded from further analysis. After removal of non-stereotypic artifacts, the data were re-referenced to common average and subjected to a temporal infomax ICA decomposition algorithm (Bell and Sejnowski, 1995), with extension to sub-Gaussian sources (Lee et al., 1999). The resulting component matrix was screened for components representing eye movement and electrode artifacts using outlier statistics and non-dipolar components (residual variance cutoff at 15\%; Delorme et al., 2012), which were removed from the data using automated outlier-based statistics (Dutra et al., 2018; Wessel, 2018a,b; Waller et al., 2019). The remaining components were subjected to further analyses. For all statistical analyses and all plots (except the topographical plots in Figs. 1a, 2a), the data were subsequently transformed to a reference-free montage using the current-source density method (Perrin et al., 1989; Tenke and Kayser, 2005), which minimizes the effects of volume conduction on the scalp-measured activity. This was done to enable $\beta$-event detection at frontocentral electrode FCz and lateral sensorimotor electrodes $\mathrm{C} 3$ and $\mathrm{C} 4$ while reducing the chance of crosscontamination by either side.

\section{$\beta$-burst detection}

$\beta$-burst detection was performed exactly as described by Shin et al. (2017). The description is adapted from therein.

First, each electrode's data were convolved with a complex Morlet wavelet of the form:

$$
w(t, f)=A \exp \left(\frac{t^{2}}{2 \sigma_{t}^{2}}\right) \exp (2 i \pi f t),
$$

with $\sigma=\frac{m}{2 \pi f}, A=\frac{1}{\sigma_{t}} \sqrt{2 \pi}$, and $m=7$ (cycles) for each of 15 evenly spaced frequencies spanning the $\beta$-band (15-29 Hz). Time-frequency power estimates were extracted by calculating the squared magnitude of the complex wavelet-convolved data. These power estimates were then epoched relative to the events in question (ranging from -500 to +1000 
ms with respect to Stop-/Go-signals). Individual $\beta$-bursts were defined as local maxima in the trial-by-trial $\beta$-band time-frequency power matrix for which the power exceeded a set cutoff of $6 \times$ the median power of the entire time-frequency power matrix for that electrode. Local maxima were identified using the MATLAB function imregional().

\section{Experimental design and statistical analysis}

Behavioral analysis. Means were extracted for each subject for the following measures: Go-trial reaction time, failed Stop-trial reaction time, Stop-signal delay, Stopping accuracy, and Stop-signal reaction time, which was calculated via the integration method with replacement of miss-trial reaction times (Verbruggen et al., 2019). Failed Stop-trial reaction times and Go-trial reaction times were compared with a paired samples $t$ test.

Topographical distribution of $\beta$-bursts. To visualize the topographical distribution of $\beta$-bursts with respect to Stop- and Go-signals, 12 windows of $25 \mathrm{~ms}$ length, starting at $25 \mathrm{~ms}$ after the event, were defined. For each subject, the number of $\beta$-bursts in each window at each electrode following the respective stimulus (Go/Stop) was counted. The average number of $\beta$-bursts in each time window for each of the three trial types (Correct Go, Successful Stop, Failed Stop) was then plotted in a topographical grid representing the scalp surface (see Figs. $1 a, 2 a$ ). Figure 3 depicts the difference between the number of $\beta$-bursts on Go- and Stoptrials in each window and at each electrode, tested for significance using paired-samples $t$ tests. The resulting electrode $\times$ time window matrix of $p$ values was corrected for multiple comparisons to a significance level of $p=0.0001$ using the false-discovery rate (FDR) procedure (Benjamini et al., 2006).

Temporal development of $\beta$-bursts. To visualize the temporal development of $\beta$-bursts on Go-trials at the two electrodes of interest (C3 and C4), 11 windows of $50 \mathrm{~ms}$ length ranging from $25 \mathrm{~ms}$ post-event to 575 $\mathrm{ms}$ post-event were defined. This time range spanned the entire post-Gosignal period leading up to (and including) mean reaction time. To test the linear decreasing trend observed at $\mathrm{C} 3 / \mathrm{C} 4$ during movement initiation for significance, we submitted the means for left- and right-hand responses to the Mann-Kendall test. To test the lateralization of the linear trend toward the end of the response period (i.e., before mean RT), we compared the means for left- and right-hand responses at both electrodes using paired-samples $t$ tests, again corrected for multiple comparisons to a significance level of $p=0.0001$ using the FDR procedure. To visualize the temporal development of $\beta$-bursts on Stop-trials at the electrode of interest $(\mathrm{FCz})$, six windows of $50 \mathrm{~ms}$ length ranging from 25 $\mathrm{ms}$ post-event to $325 \mathrm{~ms}$ post-event were defined. This time range spanned the entire post-Stop-signal period leading up to (and including) Stop-signal reaction time (SSRT; see Figs. $1 b, 2 b, d$ ).

Correlation between lateral sensorimotor $\beta$-bursts and behavior. The relative rates of $\beta$-bursts over lateral sensorimotor sites C3 and C4 for each subject and in each time-window following the Go-signal (see Fig. $1 b, d)$ were then correlated with each subject's SSRT and Go-trial reaction time using Pearson's correlation coefficient. The resulting correlations were tested for significance and the respective $p$ values were corrected for multiple comparisons using the FDR procedure (12 tests each, critical $p<0.05)$.

Comparison of pre-SSRT $\beta$-events. To test the difference in the amount of $\beta$-bursts at fronto-central electrode FCz for significance, we counted the number of $\beta$-bursts in the time period ranging from the Stop-signal to each individual participant's SSRT estimate, separately for successful and failed Stop-trials. Moreover, we counted the number of $\beta$-bursts in a time period of identical length on Go-trials. The time period of interest on Go-trials was the time period ranging from the current Stop-signal delay on that trial and the participants' SSRT estimate. In other words, it corresponded to the Stop-signal-to-SSRT time period had there been a Stop-signal on that trial. The mean number of $\beta$-bursts for these periods were compared across subjects using a paired-samples $t$ test.

Lateralized $\beta$-bursting after fronto-central $\beta$-bursts. To investigate the pattern of $\beta$-bursting over bilateral electrodes $\mathrm{C} 3 / 4$ following fronto-central $\beta$-bursts in the Stop-Signal-to-SSRT period on successful Stop-trials, we identified each succesful Stop-trial in which such a fronto-central $\beta$-burst event occurred, and counted the amount of $\beta$-bursts at electrodes C3/4 (both contralateral and ipsilateral to the to-be-stopped response) in eight time windows of $25 \mathrm{~ms}$ duration ranging from -100 to $+100 \mathrm{~ms}$ around the fronto-central $\beta$-burst event (see Fig. 4) In case more than one $\beta$-burst event was found, we chose the latency of the first of those events. To compare these $\beta$-burst counts to trials in which no fronto-central $\beta$-burst was found, a random time point in the Stop-signal-to-SSRT interval was chosen from a uniform distribution and C3/4 $\beta$-bursts were quantified in an identical time window around that random time point in the pre-SSRT period. The distribution of randomly selected time points did not significantly deviate from the distribution of $\beta$-burst onset times on trials with such bursts. We then compared the mean burst-counts for the two conditions in the four time-windows following the event (or the "pseudo-event" in the case of the trial without an actual fronto-central burst) using signed-rank tests (the non-parametrical equivalent of the paired-samples $t$ test, chosen because of the large skew of the means toward 0 in these samples), corrected for multiple comparisons to a critical $p$ value of $p<0.0001$ using the FDR method.

$\beta$-power amplitude analysis. To conduct the analyses presented in Figure 5 , the time-frequency power estimates that were used for $\beta$-burst detection (see section "Beta burst detection") were converted to decibels using a $250 \mathrm{~ms}$ baseline before the event in question (Go-signal/Stopsignal). Activity in a specific post-event time period was then defined not by the amount of individual $\beta$-bursts within in the period, but instead by the average amplitude in that same period. All other analyses were then performed in the same manner as the $\beta$-burst analyses described.

Lagged phase coherence analysis. Lagged phase coherence was quantified as described by Fransen et al. (2015). Computations were made in a beta version of the FieldTrip software package (Oostenveld et al., 2011; RRID:SCR_004849). Cycle number was set to 3 and frequencies between 8 and $35 \mathrm{~Hz}$ were chosen to cover both the $\beta$ band and the surrounding frequencies. Lagged coherence was computed on the epoched Stop-trial data for electrode FCz and on the epoched Go-trial data for electrodes C3 and $\mathrm{C} 4$.

\section{Results}

\section{Both movement initiation and cancellation are accompanied} by systematic spatiotemporal patterns of $\boldsymbol{\beta}$-bursts

Figure $1 a$ shows that after Go-signals (which prompt the start of movement initiation), bilateral sensorimotor sites (peaking over electrodes C3 and C4) initially showed localized $\beta$-bursting, which subsequently decreased in the time period leading up to movement execution. This resulted in a significant downward linear trend across the entire time period depicted in Figure $1, b$ and $d$ (linear trend for left-hand responses at C4: $Z=-3.63, p=$ 0.00028 , left-hand responses at C3: $Z=-3.22, p=0.001$, righthand responses at $\mathrm{C} 4: Z=-2.81, p=0.005$, right-hand responses at C3: $Z=-3.23, p=0.0013)$. Furthermore, this pattern lateralized toward the end of the response period, with sites contralateral to the response hand showing a stronger sustained reduction in $\beta$-bursting (significant lateralization at $p<$ 0.0001, FDR-corrected, at time five consecutive windows from 325 to $575 \mathrm{~ms}$ for electrode $\mathrm{C} 4, t_{(233)}=-4.91, p=1.75 \times 10^{-06}$, $d=0.38, t_{(233)}=-8.25, p=1.2 \times 10^{-14}, d=0.63, t_{(233)}=$ $-6.74, p=1.24 \times 10^{-10}, d=0.52, t_{(233)}=-6.83, p=7.37 \times$ $10^{-11}, d=0.61, t_{(233)}=-5.75, p=2.81 \times 10^{-08}, d=0.5$; and at three consecutive time windows from 325 to $475 \mathrm{~ms}$ for electrode $\mathrm{C} 3, t_{(233)}=5.28, p=2.92 \times 10^{-07}, d=0.41, t_{(233)}=6.02, p=$ $\left.6.6 \times 10^{-09}, d=0.49, t_{(233)}=4.81, p=2.65 \times 10^{-06}, d=0.37\right)$. These findings parallel reports from trial-averaged power-based quantifications of sensorimotor $\beta$-band activity (e.g., McFarland et al., 2000; Pfurtscheller et al., 2003). An inspection of individual trial data showed that the single-trial $\beta$-band signal was indeed characterized by clearly visible, burst-like events, rather than by steady modulations (Fig. 1c,e show data from one representative subject; plots of single trial data for each individual participant can be downloaded from https://osf.io/v3a78/). 
a Topographical distribution of $\beta$-bursts following GO-signals

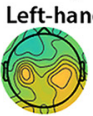

25-50
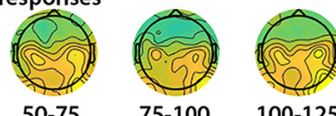

100-125

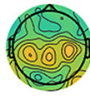

$125-150$

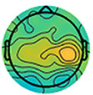

150-175

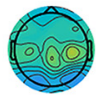

$175-200$

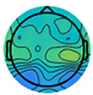

200-225

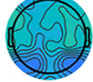

225-250

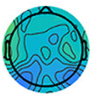

250-275

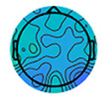

275-300

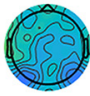

300-325
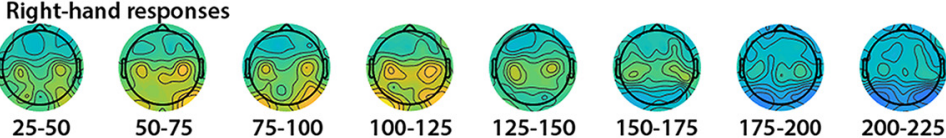

150-175

175-200

100-125

b Temporal distribution at RIGHT motor electrode C4

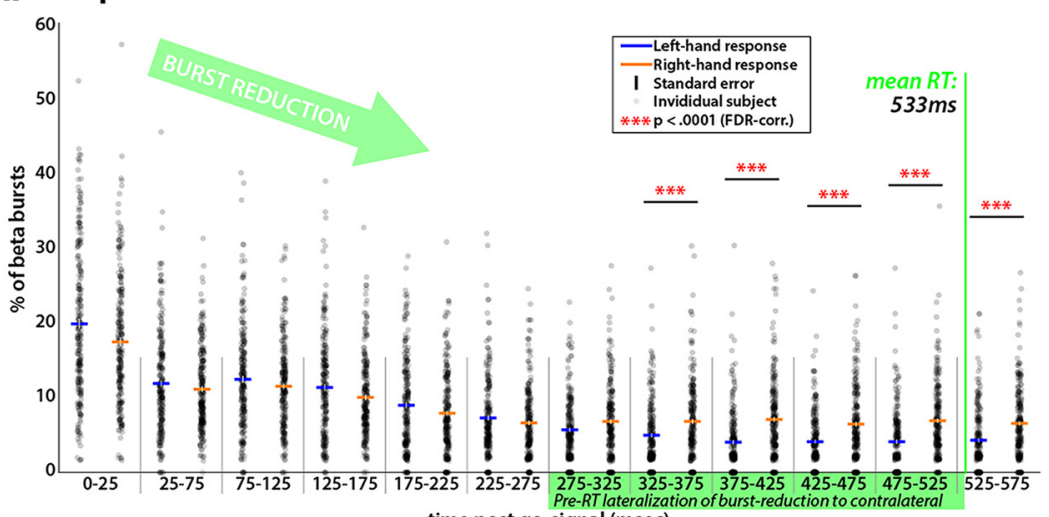

time post go-signal (msec)

d Temporal distribution at LEFT motor electrode C3

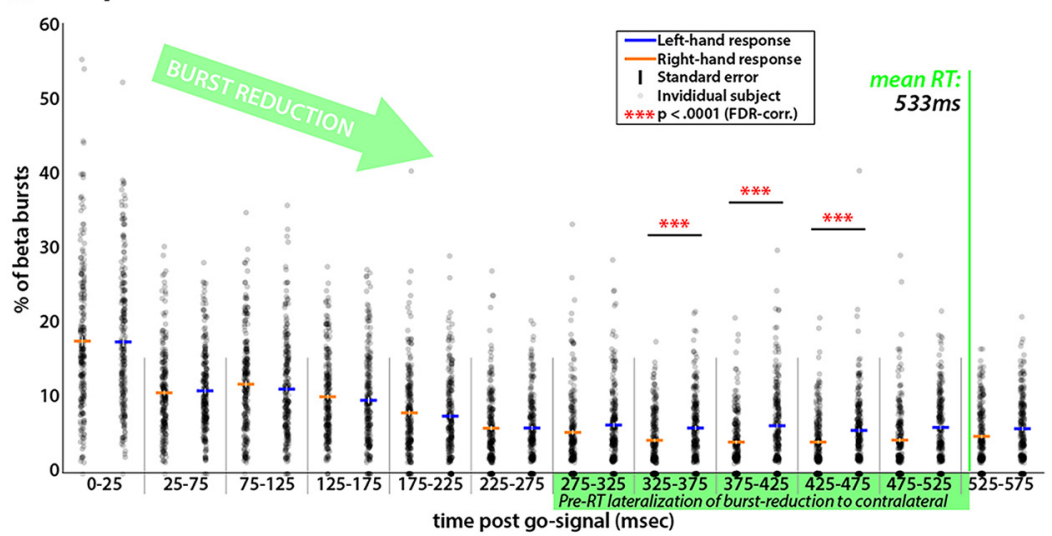

C C4: Individual trial data
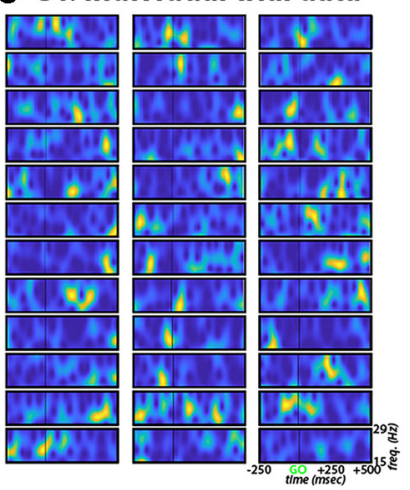

e C3: Individual trial data

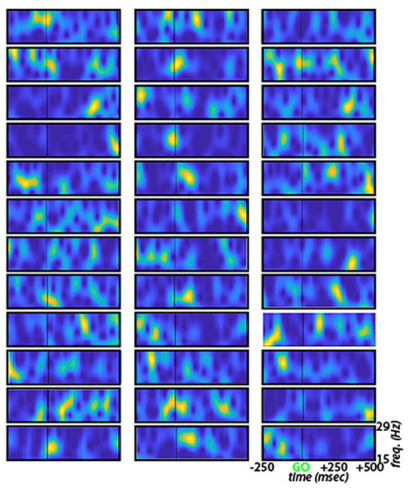

f Relationship between Go-signal-locked contralateral $\mathrm{C} 3 / \mathrm{C} 4 \boldsymbol{\beta}$-bursts and $\mathrm{GO} / \mathrm{STOP}$ behavior
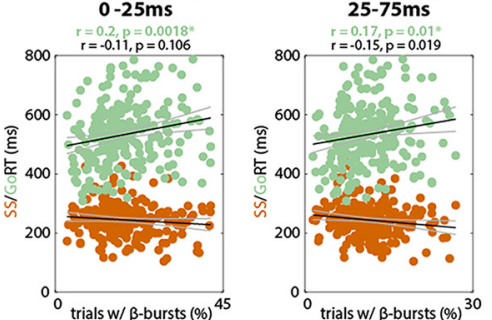

275-325ms

$800 \quad r=0.07, p=0.3083$

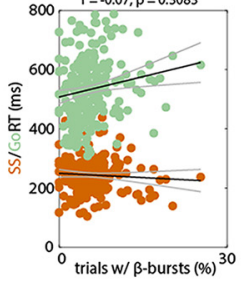

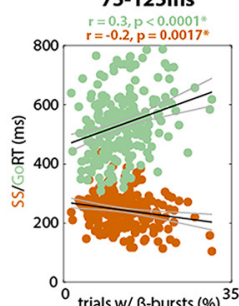
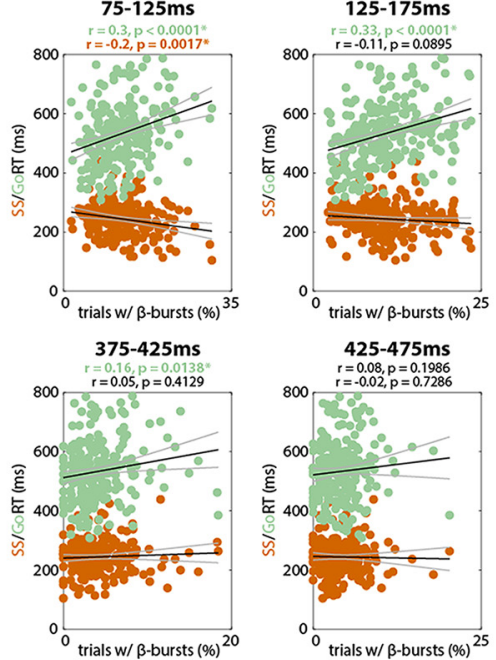

425-475ms

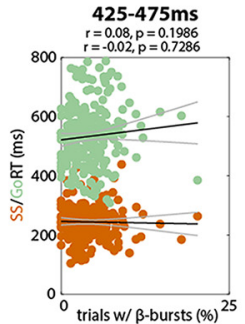

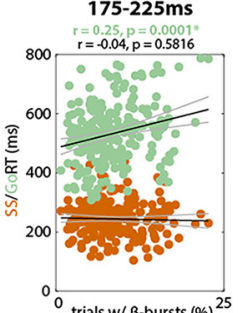
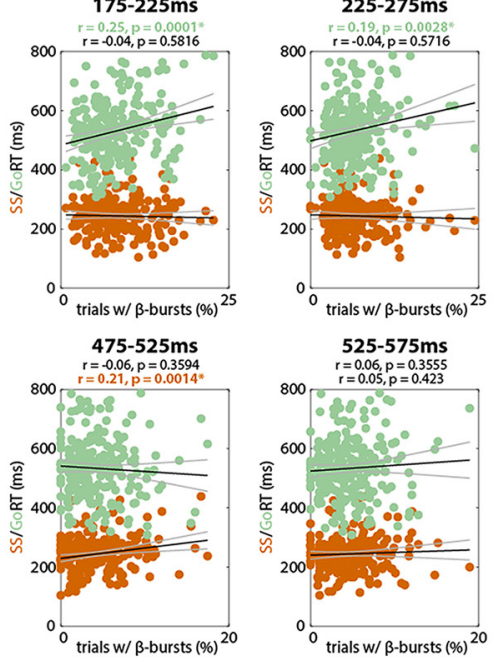

Figure 1. $\beta$-burst properties during movement initiation on Go-trials. $\boldsymbol{a}$, Topographical distribution of the average number of $\beta$-bursts on the scalp in consecutive time-windows following the Go-signal, for both left-hand (top) and right-hand (bottom) responses. There are bilateral peaks over electrode sites $(3$ and C 4 until $\sim 200$ ms following the Go-signal. Channels at which the number of bursts did not exceed significant increases from zero (when corrected using FDR) were set to $0 . \boldsymbol{b}$, Temporal development of $\beta$-burst rates following the Go-Signal at right lateral sensorimotor electrode site (4 during both left- and right-hand responses. A significant linear trend is evident, such that the $\beta$-burst rates steadily decreased (Figure legend continues.) 


\section{a Topographical distribution of $\beta$-bursts following STOP-signals}

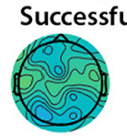

25-50

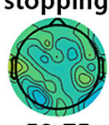

50-75
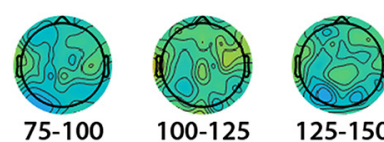

125-150

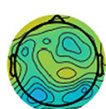

150-175

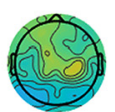

$175-200$

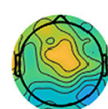

200-225

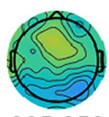

225-250 : $245 \mathrm{~ms}$

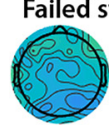

$25-50$

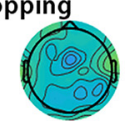

50-75
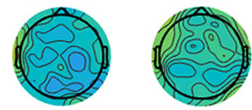

100-125

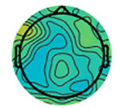

125-150

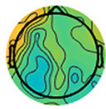

150-175

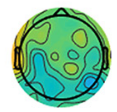

175-200

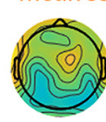

200-225

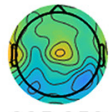

225-250

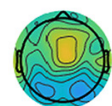

250-275

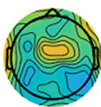

250-275
275-300
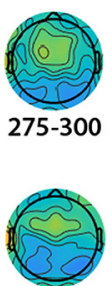

275-300

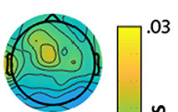

300-325

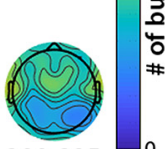

\section{b Temporal distribution at electrode $\mathrm{FCz} \quad \mathbf{c}$ FCz $\beta$-bursts before SSRT}
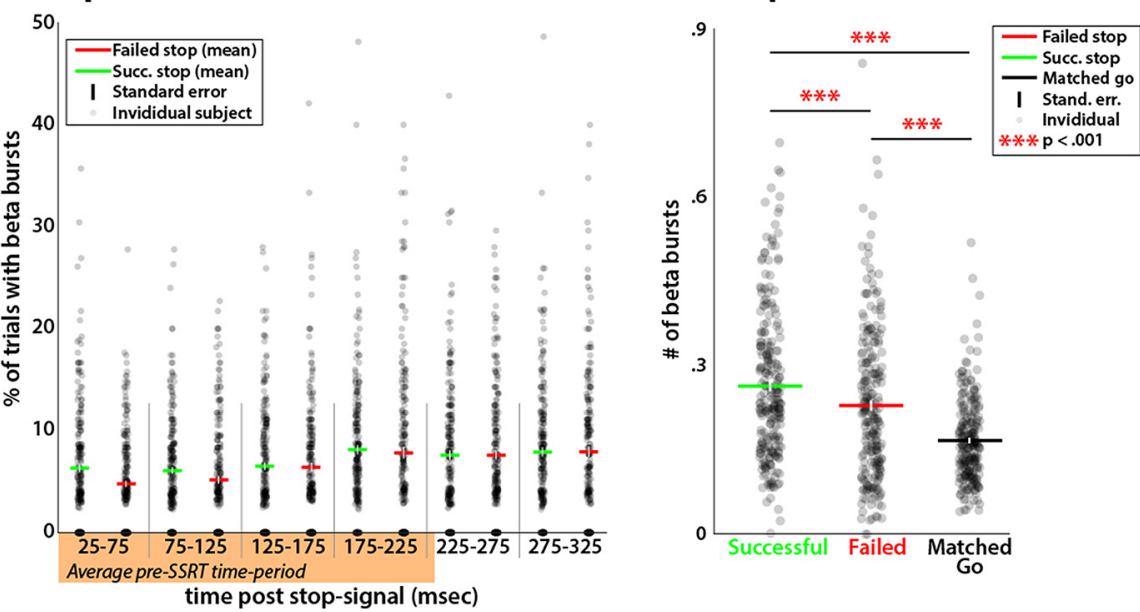

\section{d Individual trial data at $\mathrm{FCz}$}

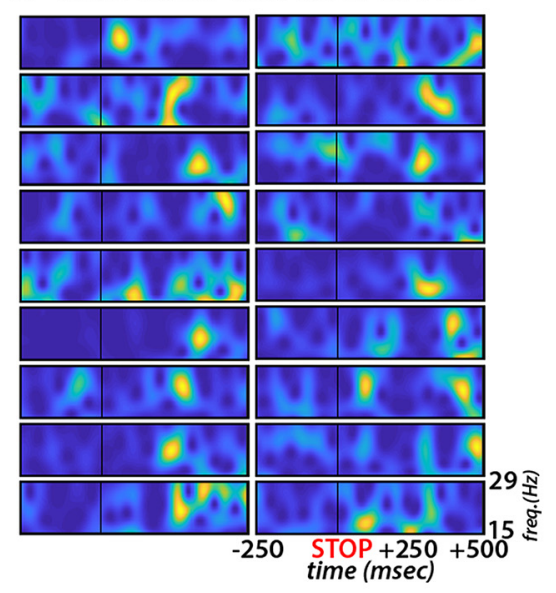

Figure 2. $\quad \beta$-burst properties during movement cancellation on Stop-trials. $\boldsymbol{a}$, Topographical distribution of the number of $\beta$-bursts on the scalp in consecutive time-windows following the Stop-signal, separately for successful (top) and failed (bottom) Stop-trials. In the time-window toward the end of SSRT, a clear fronto-central organization of $\beta$-bursting centered around electrode $\mathrm{FC} z$ is evident. Channels at which the number of bursts did not exceed significant increases from zero (when corrected using FDR) were set to 0 . $\boldsymbol{b}$, Temporal development of $\beta$-burst rates following the Stop-Signal at fronto-central electrode FCz.c,Comparison of the number of $\beta$-bursts between successful and failed Stop-trials in the Stop-signal-to-SSRT period (as well as during a matched time period on G0-trials) for each subject. $\boldsymbol{d}, \beta$-band data at electrode $\mathrm{FC}$ from 18 individual Stop-trials in a representative subject, showing the burst-like nature of $\beta$-activity following the stop-signal (vertical line). Each plot shows frequency on the $y$-axis $(15-29 \mathrm{~Hz})$ and time relative to the Stop-signal on the $x$-axis, as demarcated on the bottom right trial (labels were removed from the remaining trials for easier viewing). The same plots of single trial data for each individual participant can be downloaded from the OSF URL in the Methods section.

With regards to movement cancellation, Figure $2 a$ shows that after Stop-signals (which followed Go-signals on $1 / 3$ of all trials at a variable delay and prompted the participants to attempt to cancel the movement instructed by the Go-signal), no coherent spatiotemporal organization of the rate of $\beta$-bursts could be observed until $\sim 200 \mathrm{~ms}$ after the stop-signal. At that point, a clear radial fronto-central topographical distribution emerged, centered around electrode FCz. Just like Go-signal-related activity at

$\leftarrow$

(Figure legend continued.) after the Go-signal. Moreover, a significant lateralization of this effect is evident starting at $325 \mathrm{~ms}$ following the Go-signal, such that the $\beta$-burst rates for the contralateral hand kept diminishing, while the rates showed an earlier asymptote for the ipsilateral response hand. c, $\beta$-band data at right sensorimotor electrode 44 from 36 individual Go-trials in a representative subject, showing the burst-like nature of $\beta$-activity. Each plot shows frequency on the $y$-axis $(15-29 \mathrm{~Hz})$ and time relative to the $\mathrm{G}_{0}$-signal on the $x$-axis, as demarcated on the bottom right trial (labels removed from the other trial plots for easier viewing). These same plots of single trial data for all individual participants can be downloaded from the OSF URL in the Methods section. $\boldsymbol{d}$, Same as $\boldsymbol{b}$, but for left-lateral electrode C3.e, Same as $\boldsymbol{c}$, but for C3. $\boldsymbol{f}$, Intersubject correlation between average Go-trial reaction time (green dots)/ average Stop-signal reaction time (orange dots) and each subject's $(3 / C 4 \beta$-burst rates contralateral to the instructed movement, separately for each of the time windows in $\boldsymbol{b}$. Black line, Least-squares fit; gray lines, confidence intervals. ${ }^{*} p<0.05$ (FDR-corrected). Increased amounts of contralateral $\beta$-bursting between 75 and 125 ms following the Go-signal were related to longer Go-trial reaction times, as well as shorter Stop-signal reaction times.
C3 and C4, single-trial Stop-signal-related activity at FCz clearly showed the presence of $\beta$-bursting (Fig. $2 d$ ).

Fronto-central $\beta$-bursting is increased during successful movement cancellation

Behavior in the Stop-signal task was typical for healthy young adults (mean Go-trial reaction time: $534 \mathrm{~ms}$, SEM: 6.6; mean failed Stop-trial reaction time: $460 \mathrm{~ms}$, SEM: 5.79; stop accuracy: 0.52, SEM: 0.002, mean Stop-signal delay: 282 ms, SEM: 7.91; mean Stop-Signal reaction time: 245 ms, SEM: 3.62). In accordance with the race model, failed Stop-trial reaction times were consistently faster than Go-trial reaction times: $t_{(233)}=34.7, p=$ $4.9 \times 10^{-94}, d=0.78$. On the individual-subject level, 232 of 234 participants showed this pattern.

The observed increase of fronto-central $\beta$-bursting after Stopsignals (compared with Go-signals) starting $\sim 200 \mathrm{~ms}$ following the Stop-signal was highly significant. Figure 3 shows the topographical difference plots between the distribution of $\beta$-bursts following Stop- versus Go-trials, thresholded to a family-wise $p=0.0001$ using the FDR-correction for 62 channels and 12 time windows. Moreover, the time after the Stop-signal at which this fronto-central organization of $\beta$-bursting developed overlaps with the end of SSRT ( $\sim 245 \mathrm{~ms})$. This time period, before the end of SSRT, is the exact time period during which neural activity reflecting movement cancellation should be maximal according 


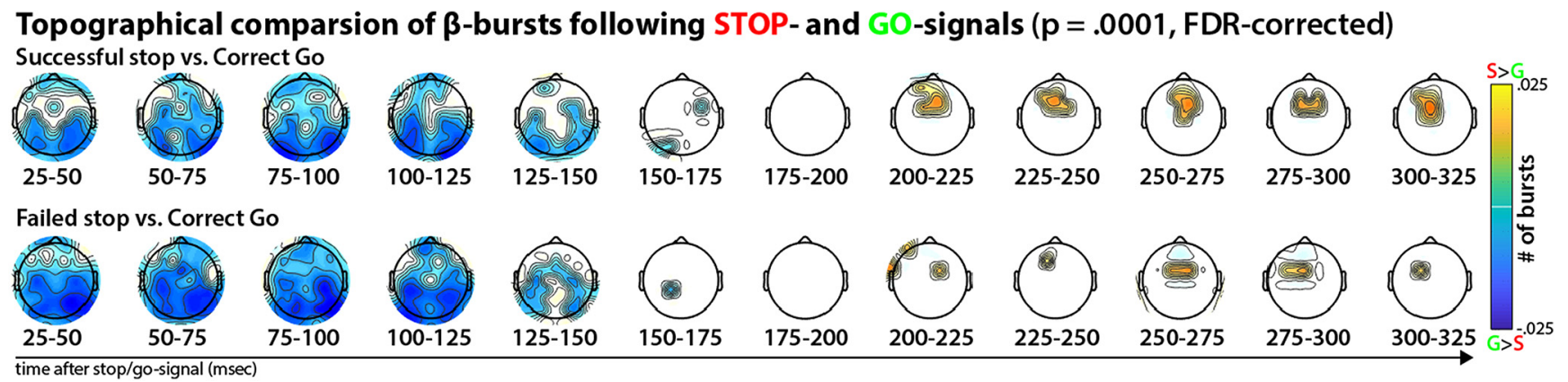

Figure 3. Statistical comparison of $\beta$-burst topographies following Stop- and Go-signals, separately for successful (top) and failed (bottom) Stop-trials. Thresholded for significance at $p<$ 0.0001 , FDR-corrected for multiple comparisons across channels and time windows. It is evident that while movement initiation (Go-trials) was accompanied by a significantly relative increase in $\beta$-burst count at bilateral sensorimotor sites until $\sim 175 \mathrm{~ms}$ after the Go-signal, movement cancellation was accompanied by significant relative increase of $\beta$-burst rates at fronto-central electrodes, starting at $\sim 200 \mathrm{~ms}$ after the Stop-signal.

to both computational models of the Stop-signal task (Boucher et al., 2007) and neural recordings from the basal ganglia and the frontal eye fields (Hanes et al., 1998; Ogasawara et al., 2018). Moreover, a direct comparison of the pre-SSRT time period in each individual participant (i.e., the time range between the appearance of the Stop-signal and the end of each participant's individual SSRT estimate) revealed that successful Stop-trials yielded an increased rate of $\beta$-bursts at FCz compared with failed Stop-trials $\left(t_{(233)}=3.46, p<0.0007, d=0.25\right.$; Fig. $\left.2 b, c\right)$ as well as compared with a matched time-period on Go-trials $\left(t_{(233)}=\right.$ $\left.12.28, p=4.8 \times 10^{-27}, d=0.88\right)$. This suggests that frontocentral $\beta$-bursts are related to movement cancellation, with successful stop-trials being accompanied by greater burst-rate increases. These findings parallel reports of increased trialaveraged $\beta$-band power found in intracranial recordings from neurological patients, where increased $\beta$-power can be found at sites in the medial wall of the frontal cortex; most notably, in the pre-supplementary motor area (Swann et al., 2012; Jha et al., 2015).

\section{Fronto-central $\beta$-bursts are followed by increased bilateral} sensorimotor $\boldsymbol{\beta}$-bursting

Figure $4 a$ shows the temporal development of $\beta$-bursting at sensorimotor sites ipsilateral and contralateral to the to-be-stopped movement on successful stop-trials. Importantly, rather than being time-locked to the Stop-signal (or the Go-signal), these plots are time-locked to the latency of the first fronto-central $\beta$-burst event that occurred within the pre-SSRT time period on successful Stop-trials (i.e., between the Stop-signal and the participant's SSRT estimate). These plots show a significant increase in bilateral sensorimotor $\beta$-bursting within $25 \mathrm{~ms}$ of the first frontocentral $\beta$-burst. To evaluate significance, these values were compared with matched time-periods on successful stop-trials that did not show fronto-central $\beta$-bursts in the pre-SSRT period (exact values for the pairwise comparisons between trials with and without $\beta$-bursts: $Z=7.81, p=5.65 \times 10^{-15}$ and $Z=4.36$, $p=1.28 \times 10^{-05}$ for the two significant time windows for contralateral sites and $Z=8.06, p=7.7 \times 10^{-16}$ and $Z=4.8, p=$ $1.55 \times 10^{-06}$ for ipsilateral sites). Additionally, these same time windows of post-burst activity were compared with the average number of sensorimotor $\beta$-bursts that occurred on trials with fronto-central bursts, but before the occurrence of that burst (i.e., the average number of bursts in the four time windows before the fronto-central $\beta$ burst on the same trials). That comparison confirmed the significant increase in sensorimotor $\beta$ bursting following the fronto-central $\beta$-burst, though in this analysis, this was limited to the first post-burst window $\left(Z=8.02, p=1.1 \times 10^{-15}\right.$ for contralateral, $Z=6.63, p=3.35 \times 10^{-11}$ for ipsilateral). Moreover, the same analysis performed on failed instead of successful stop-trials (which was suggested by a reviewer) yielded no significant increases in sensorimotor $\beta$ following fronto-central $\beta$ bursts. In essence, these findings suggest that there is a lowlatency increase in lateral sensorimotor $\beta$-bursts immediately following fronto-central $\beta$-bursts that occur within the Stopsignal-to-SSRT period on successful Stop-trials. Potential effects of volume-conduction are a concern in this analysis (though these data were current-source density transformed; see Materials and Methods). However, a topographical representation of the effect reveals that the increase in $\beta$-bursting following the first fronto-central $\beta$-burst showed local maxima at bilateral sensorimotor sites (in addition to the fronto-central electrodes surrounding FCz; Fig. 4b); i.e., at remote locations that do not follow a linear or exponential signal decay from FCz. Therefore, although volume conduction effects can never be ruled out in scalp-recordings, there seems to be some specificity of this effect to bilateral sensorimotor sites.

\section{Early $\boldsymbol{\beta}$-bursting at lateral sensorimotor sites may reflect a tonic inhibitory state}

The fact that lateral sensorimotor $\beta$-bursting steadily declined during movement initiation (Fig. $1 b, d$ ) and restarted immediately following fronto-central $\beta$-bursts during movement cancellation (Fig. 4a) suggests that lateral sensorimotor beta-bursting may reflect an inhibited motor state, which has to be overcome during movement initiation and which can be rapidly reinstated following frontal control signals during movement cancellation. To further test this possibility, we correlated the amount of contralateral sensorimotor $\beta$-bursting on Go-trials with the two main behavioral indices for each subject (Go-trial reaction time and Stop-signal reaction time). This was done for the same time windows that were used to visualize these same $\beta$-burst data in Figure $1, b$ and $d$. As can be seen in Figure $1 f$, the amount of lateral sensorimotor $\beta$-bursting was significantly positively related to Go-trial reaction times in all nine of the early time windows following the Go-signal (significant time-windows in which the correlation survived FDR-correction covered a time range from the Go-signal to $425 \mathrm{~ms}$ after the signal). The strongest correlation was found during the earlier time windows, in particular the ones ranging from 75 to $175 \mathrm{~ms}$ after the Go-signal. Specifically, subjects that showed increased rates of $\beta$-bursting during these time windows showed systematically longer Go-trial reaction times. Furthermore, the rate of these early sensorimotor 
a Increased lateralized $\boldsymbol{\beta}$-bursting after fc $\boldsymbol{\beta}$-bursts on STOP-trials CONTRALATERAL C3/C4

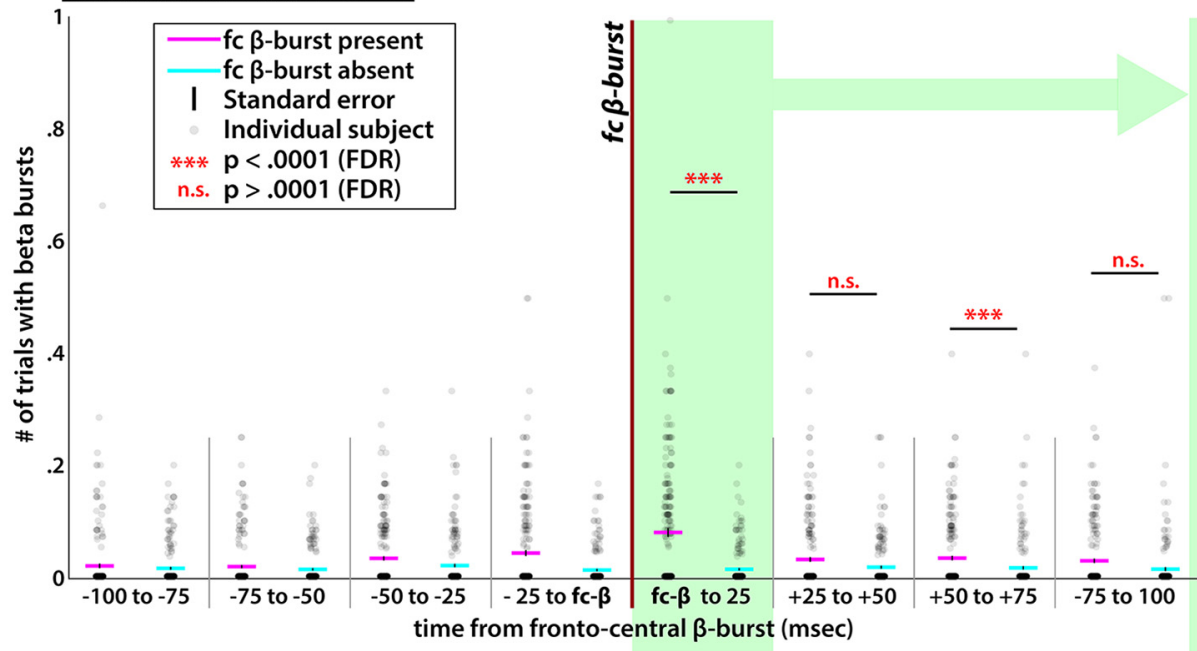

\section{IPSILATERAL C3/C4}

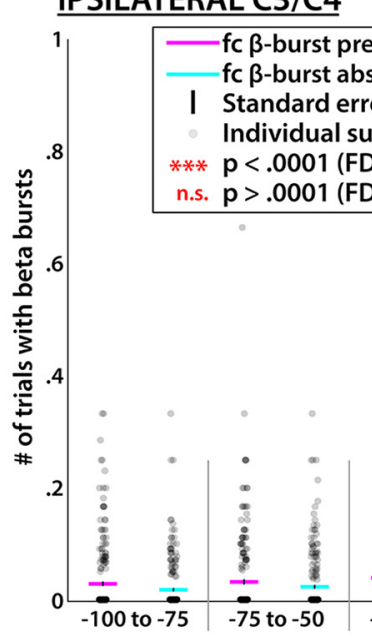

\section{b}

Topographical distribution OF $\beta$-BURSTS WITHIN 25 MSEC OF fC $\beta$-BURST

TRIALS WITH FC $\beta$-BURSTS

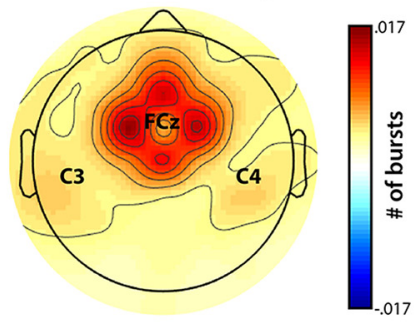

TRIALS WITHOUT FC $\beta$-BURSTS

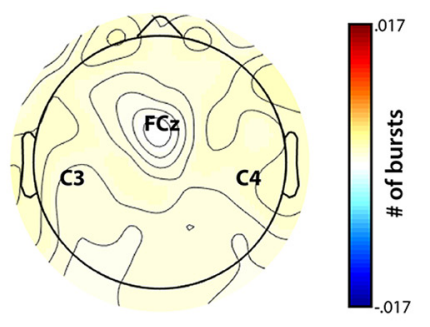

DIFFERENCE $(p<.000001, F D R)$

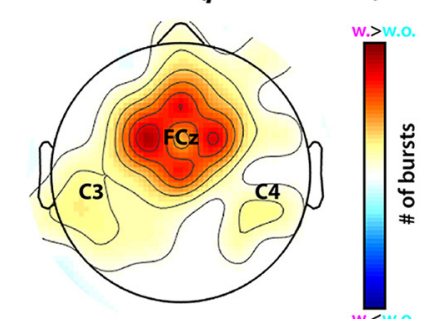

Figure 4. Interaction between fronto-central and bilateral sensorimotor $\beta$-bursting during successful movement cancellation. $\boldsymbol{a}$, Rates of contralateral and ipsilateral $\beta$-bursts at sensorimotor electrodes $\mathrm{C} 3 / \mathrm{C} 4$, time-locked to individual fronto-central $\beta$-bursts during the Stop-signal-to-SSRT period. Twenty-five milliseconds following fronto-central $\beta$-bursts (green highlighting), both sensorimotor $\beta$-bursting was significantly increased at sites both ipsilateral and contralateral to the to-be-stopped movement. $\boldsymbol{b}$, Topographical representation of $\beta$-bursts in the highlighted time period ( $25 \mathrm{~ms}$ following the fronto-central $\beta$-burst event) showing local maxima in increased $\beta$-burst activity at bilateral sensorimotor sites (in addition to the fronto-central sites that surround $\mathrm{F}(\mathrm{z})$.

$\beta$-bursting showed a negative relationship with SSRT: subjects with higher initial sensorimotor $\beta$-bursting rates showed faster SSRT. The latter relationship survived FDR-corrections for multiple comparisons in the $75-125 \mathrm{~ms}$ post-Go-signal window. Together with the Go-reaction time correlation, this suggests that the rate of early $\beta$-bursting over lateral somatosensory areas does indeed reflect a (proactive) inhibition of the motor system, which is detrimental to fast movement execution (as reflected in slower Go-trial reaction times), but beneficial to fast movement cancellation (as reflected in faster Stop-signal reaction times).

\section{$\boldsymbol{\beta}$-burst rates are a more accurate level of description compared with mean amplitude changes}

As is evident from the individual-trial plots in Figures $1, c$ and $e$, and $2 d$ (as well as from prior literature), raw, single-trial betaband activity is characterized by transient, burst-like events, rather than slow and steady modulations of overall power. However, it is an open question whether $\beta$-bursts actually provide a more accurate description of the relationship between human brain activity and motor behavior especially compared with more standard mean-amplitude measurements. To investigate this question, two of the three main analyses in the current paper [(1) the comparison of fronto-central $\beta$-activity between the different trial types, Fig. $2 c$; and (2) the correlation between sensorimotor $\beta$-activity and Go-/Stop-signal reaction time, Fig. $1 f]$ were repeated using standard mean amplitude measurements. (The third main finding in this current study, i.e., the increase in $\beta$-bursting at sensorimotor sites following individual instances of fronto-central $\beta$-burst events, cannot be reproduced using amplitude measurements.) In other words, instead of quantifying $\beta$-burst rates for the time periods of interest, overall $\beta$-amplitude activity was averaged across the same period of time, and the analyses were repeated just as presented above.

Figure $5 a$ shows the thresholded topographical distribution of mean $\beta$-amplitude across the time periods used in the $\beta$-burst analyses in Figures 1, 2, and 3. From the statistical trial-comparison maps (Fig. $5 a$, bottom two rows, i.e., the analog of Fig. 3 ), it is evident that $\beta$-band amplitude shows topographically similar 


\section{a Topographical distribution of $\beta$-amplitude per trial type}

Correct Go-trials

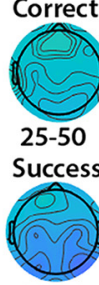

25-50

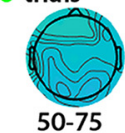

50-75
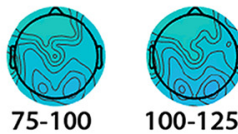

75-100

100-125
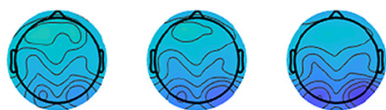

125-150

150-175

$175-200$
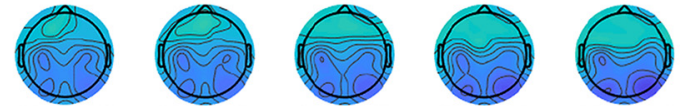

100-125

125-150

150-175

175-200

200-225
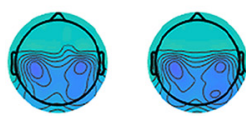

100-125

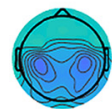

125-150

150-175

175-200
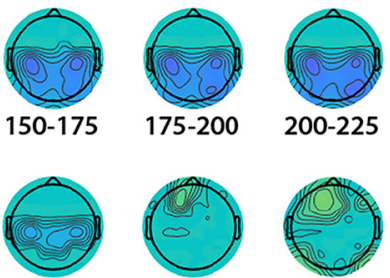

200-225

150-175

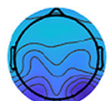

200-225

200-225

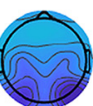

225-250

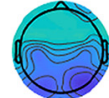

225-250

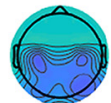

225-250

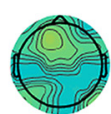

225-250

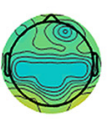

200-225

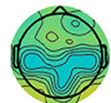

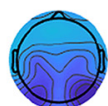

250-275
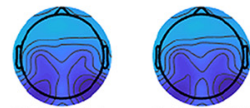

275-300

300-325

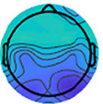

250-275
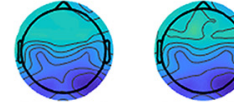

275-300

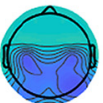

250-275

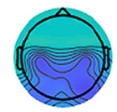

275-300
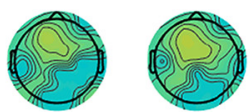

275-300

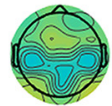

250-275

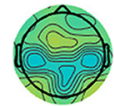

275-300

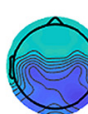

300-325

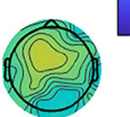

300-325

Failed Stop vs. Correct Go

$$
25-50 \quad 50-75
$$

\section{b Mean $\beta$-amplitude at $\mathrm{FCz}$}

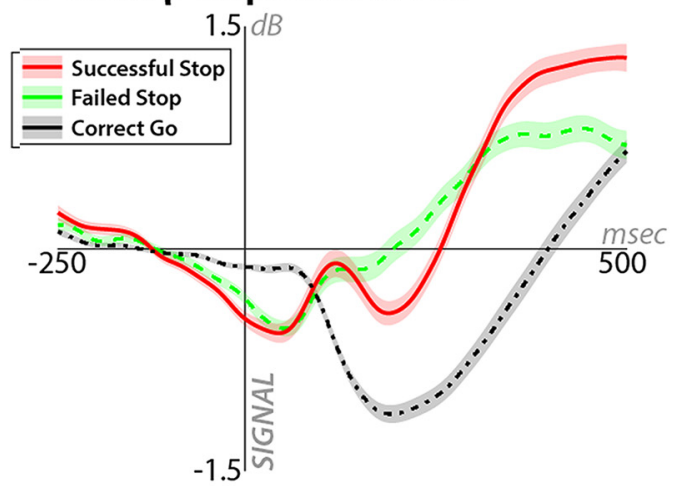

\section{C $\beta$-ampl. pre-SSRT}

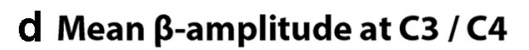

d Mean $\beta$-amplitude at C3 / C4
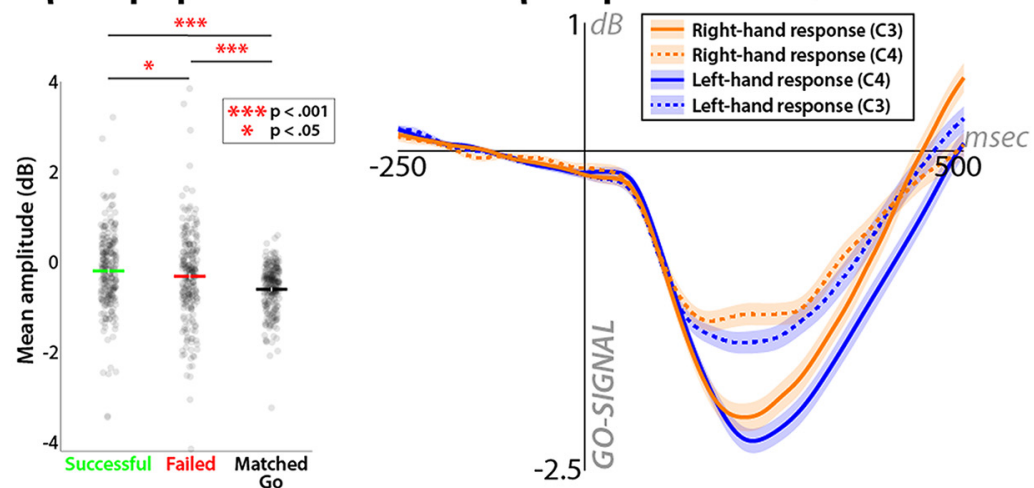

e Relationship between Go-signal-locked contralateral C3/C4 $\beta$-amplitude and GO/STOP behavior
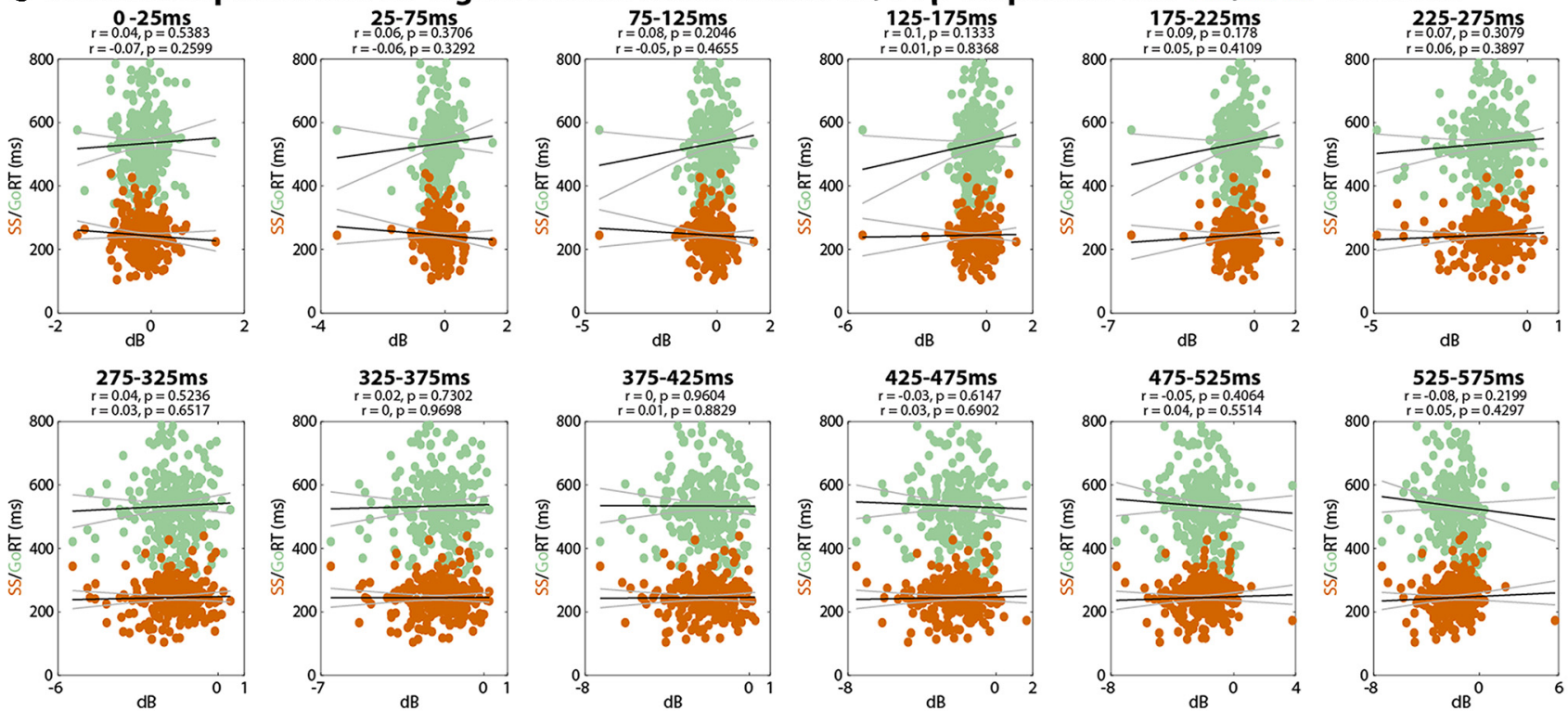

Figure 5. $\beta$-power amplitude analyses. $\boldsymbol{a}$, Topographical distributions of $\beta$-power amplitude following $\mathrm{G} 0$ - and Stop-signals. These figures are analog to Figures $1 a, 2 a$, and 3 , except they do not show changes in $\beta$-burst rates, but changes in mean $\beta$-amplitude during the same time windows. Any activity that did not exceed a significant change from $0 \mathrm{~dB}$ (using the same significance threshold of $p<0.0001$, FDR-corrected that was used in Figs. 1a, 2a, and 3) was set to $0 . \boldsymbol{b}$, Changes in mean $\beta$-power amplitude at fronto-central electrode $\mathrm{FCz}$ on the three trial types of interest in the Stop-signal task. Shaded area around each curve represents the SEM. c, Comparison of mean $\beta$-amplitude in the Stop-signal-to-SSRT period (and a matched time (Figure legend continues.) 

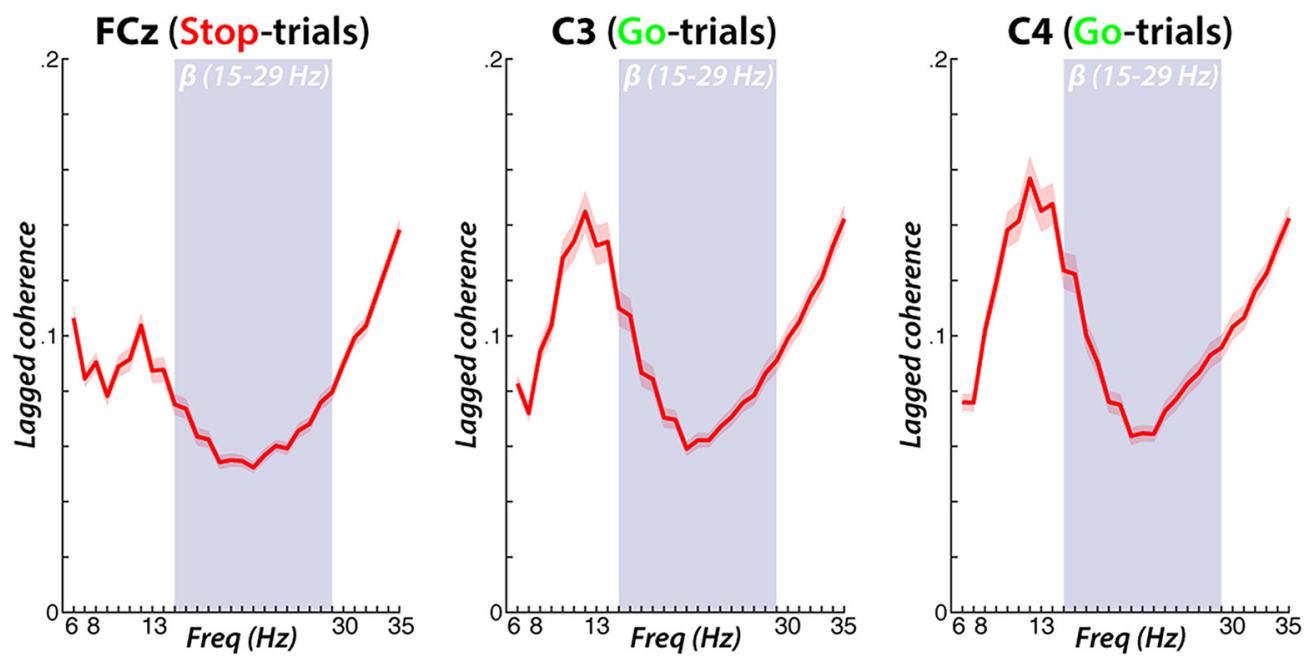

Figure 6. Lagged phase coherence ( 3 cycles) at the three electrode sites of interest at frequencies including and surrounding the $\beta$-range. What can be seen is that the $\beta$-range (highlighted) contains the trough of the lagged coherence spectrum, suggesting that the $\beta$ signal at those electrodes is more burst-like than the signal in the surrounding frequencies.

features compared with $\beta$-burst-rates: there is bilateral sensorimotor desynchronization following Go-signals and a frontocentral power increase following Stop-signals. However, unlike for $\beta$-burst rates, where fronto-central electrode sites showed significant increases from zero following Stop-signals, the $\beta$-band power increase at fronto-central electrodes on those same trials was not significantly different from zero (Fig. $5 a$, compare second and third rows).

The average time course of the fronto-central amplitudesignal for each individual trial type can be seen in Figure $5 b$. An analysis of the $\beta$-amplitude data during the Stop-signal-to-SSRT time period (as well as a matched time period on Go-trials) revealed that, just like for the $\beta$-burst analyses, there was a significant increase of $\beta$-activity on successful compared with failed Stop-trials $\left(t_{(233)}=2.09, p=0.037, d=0.12\right)$, as well as compared with Go-trials Go-trials $\left(t_{(233)}=7.81, p=1.93 \times 10^{-13}\right.$, $d=0.55$; Fig. $5 c$ ). However, the effect sizes for both comparisons were substantially reduced compared with the $\beta$-burst analysis (indeed, the 95\% confidence interval for the effect size of the successful vs failed Stop-trials contrast included an effect size of 0 , which was not the case for the $\beta$-burst analysis).

The time course of the sensorimotor signal on Go-trials can be seen in Figure $5 d$. As to be expected based on prior work, there was a pronounced $\beta$-desynchronization following the Go-signal, which lateralized toward the contralateral side in the lead-up to the response. Although this also matches the properties of the $\beta$-burst rates reported in Figure $1, b$ and $d$, the same correlations analyses that yielded highly significant relationships between $\beta$-burst rates and both Go- and Stop-signal reaction time (Fig. 1f) did not yield any significant relationships between mean $\beta$-power amplitude and behavior. Not only did none of the 12 time periods yield any correlations that survived corrections for multiple comparisons, but none of the windows showed even an uncorrected $p<0.05$ (Fig. 5e).

(Figure legend continued.) period on Go-trials); analog to Figure 2c. d, Changes in mean $\beta$-power amplitude at sensorimotor electrodes $\mathrm{C} 3$ and $(4$ following $\mathrm{Go}$-signals on left/righthand Go-trials. Shaded area around each curve represents the SEM. $\boldsymbol{e}$, Correlations between mean $\beta$-power amplitude at sensorimotor sites contralateral to the response hand on Go-trials in 12 successive time windows following the Go-signal and Go/Stop-behavior (Go-trial reaction time/Stop-signal reaction time); analog to Figure $1 f$.
Together, these results show that while $\beta$-burst rates and amplitude changes are clearly related both theoretically (a $\beta$-burst represents a sudden, short-lived increase in $\beta$-power) and empirically (the overall topographies of both $\beta$-burst rates and $\beta$-power after Stop- and Go-signals are morphologically similar), $\beta$-burst rates not only provide a superior description of the signal on the trial-to-trial level, they also provide a much stronger relationship to behavior; and can in fact be used to uncover neuralbehavioral associations that are absent in averaged $\beta$-power data.

\section{Is single-trial $\boldsymbol{\beta}$ truly burst-like?}

One issue that bears discussion regarding the current paper (as well as the existing $\beta$-burst literature) is that the judgment of whether the $\beta$-band signal is truly burst-like (or, instead, reflects a transient modulation of an ongoing oscillation) is made based on largely qualitative, subjective criteria (van Ede et al., 2018). In other words, $\beta$ is deemed "burst-like" because the data look burst-like; and because there are concrete biophysical models that explain the burst-like nature of the $\beta$ signal (Sherman et al., 2016), which provide a priori credence to this idea.

However, one way to actually quantify whether a neural signal is truly burst-like is lagged phase coherence (Fransen et al., 2015). Lagged phase coherence quantifies the phase consistency in successive segments of data. The assumption is that if a signal at a specific frequency is marked by an ongoing oscillation, it should be possible to predict the phase of a given data segment based on past segments. Hence, lagged phase coherence was quantified at the three main electrode sites of interest in the current study (FCz, C3, C4). Figure 6 shows the lagged coherence spectrum. It is evident that although there is some degree of lagged coherence across all frequencies surrounding (and including) $\beta$, the $\beta$ band notably represents the trough of the spectrum and is flanked by higher relative degrees of coherence in both the $\alpha$ and $\gamma$ bands. Although there is currently no way to quantify what constitutes a "significant" degree of lagged phase coherence (Fransen et al., 2016), it is save to conclude that beta is at least less "oscillatory", and hence, more burst-like, than the surrounding frequency bands.

\section{Discussion}

Human movement is accompanied by systematic spatiotemporal changes in $\beta$-bursting. Before movement initiation, bilateral sen- 
sorimotor sites showed tonic, local $\beta$-bursting, potentially representing a net-inhibited state of the motor system (Engel and Fries, 2010; Picazio et al., 2014; Rossiter et al., 2014). This $\beta$-bursting steadily reduced during movement initiation, suggesting netdisinhibition. Notably, this reduction lateralized just before movement execution; comparable to the movement-related lateralization of $\beta$-desynchronization in the trial-average (McFarland et al., 2000; Kaiser et al., 2001; Doyle et al., 2005). However, $\beta$-bursts contained systematic relationships to both movement execution and movement cancellation that were absent in the averaged $\beta$-power amplitudes.

In situations in which this state of inhibition had to be rapidly reinstated, i.e., to cancel movements following Stop-signals, $\beta$-burst rates were first significantly increased at fronto-central sites. This increase showed its most coherent spatiotemporal organization during the time period toward the end of SSRT (Fig. $1 a)$. Such fronto-central $\beta$-bursts were then followed by a rapid, low-latency $(<25 \mathrm{~ms})$ increase of $\beta$-bursting over sensorimotor sites; both ipsilateral and contralateral to the to-be-cancelled movement. We tentatively propose that this may reflect a lowlatency re-instantiation of inhibition at the level of the motor system (tonic lateral sensorimotor $\beta$-bursting), triggered by a fronto-central control signal (phasic fronto-central $\beta$-bursts).

The $\beta$-burst patterns show intriguing conceptual and empirical overlap with the proposed properties of the neural and behavioral processing-cascade underlying movement cancellation. First, $\beta$-band power increases are routinely found in trialaveraged activity during movement cancellation (Swann et al., 2009, 2011, 2012; Ray et al., 2012; Wagner et al., 2018), including in intracranial recordings from sites that could conceivably underlie the fronto-centrally distributed pattern of $\beta$-bursting observed here (e.g., the pre-supplementary motor area; Swann et al., 2012). The current results suggest that these changes may actually result from increases in $\beta$-burst rates on individual trials. Second, the race-model of the Stop-signal task proposes that actionstopping can be modeled by a race between two processes; a Go-process working toward the execution of the motor response and a Stop-process working toward the cancellation of that response (Logan and Cowan, 1984). Notably, it is still controversial whether these two processes operate independently, or whether the Stop-process directly influences the Go-process (Boucher et al., 2007; Verbruggen and Logan, 2008; Schmidt et al., 2013; Schall et al., 2017). In our data, stopping-related $\beta$-bursts at fronto-central sites were immediately followed by a re-instantiation of $\beta$-bursting over sensorimotor sites; the same signature whose initial reduction marked the start of the Go-process. This suggests that the initiation of the Stop-process is followed by a substantial alteration in the neural activity underlying the Goprocess; speaking in favor of an interactive race-model (Boucher et al., 2007; Schall et al., 2017). Third, previous research has shown that humans proactively inhibit their movement initiation when they anticipate having to potentially cancel an action, resulting in longer Go-trial reaction times. The degree of this proactive inhibition is inversely related to the amount of reactive inhibition necessary to successfully cancel an action. In other words, subjects that exert higher degrees of proactive Go-trial reaction time slowing in turn exhibit shorter SSRTs (Chikazoe et al., 2009; Verbruggen and Logan, 2009). The degree of such proactive inhibitory control may be reflected in the level of initial bilateral sensorimotor $\beta$-bursting found in individual participants: Figure $1 f$ shows that higher initial sensorimotor $\beta$-burst rates predicted both slower Go-trial reaction times and faster SSRT. This suggests that early $\beta$-bursting may reflect the tradeoff between proactive control and reactive control, with more cautious responding being reflected in increased bilateral $\beta$-bursting. Fourth, the Stop-related re-instantiation of sensorimotor $\beta$-bursting that was observed following fronto-central $\beta$-bursts was notably present at both ipsilateral and contralateral sensorimotor sites (Fig. 4). This parallels prior work using physiological measures of motor excitability in specific corticospinal tracts. Such studies found that rapid movement cancellation is nonselective and affects the entire motor system (Badry et al., 2009; Majid et al., 2012; Wessel et al., 2013; Duque et al., 2017). The simultaneous, bilateral reactivation of sensorimotor $\beta$-bursting after fronto-central Stop-related $\beta$-bursts observed here could be the neurophysiological expression of that same nonselective property.

Beyond this theoretical and conceptual coherence with prior work, the quantification of $\beta$-bursts also substantially extends our insights into the neural dynamics of movement. $\beta$-bursts not only provide a more accurate description of the actual raw $\beta$-signal (compare Figs. $1 c, e, 2 b$ ), but reveals relationships to behavior that are not evident in amplitude-averages; such as the relationship between early sensorimotor activity and Go-/Stopsignal reaction time. Moreover, trial-level comparisons of $\beta$-burst rates yielded much larger effect sizes compared with amplitude analyses. For example, the effect size for the frontocentral successful versus failed Stop-trial comparison was twice as large for $\beta$-burst rates compared with mean amplitudes, suggesting that averaging partially obscures the nature of this relationship. Finally, the $\beta$-burst-locked analysis presented in Figure 4 yielded fundamentally novel insights into the interactions between neural concomitants of the Go- and Stop-processes and their interaction during movement cancellation. Notably, the current study could speak to another recent controversy in the field of inhibitory control. Some studies have suggested that SSRT overestimates the latency of the stopping process (Matzke et al., 2013), and that true indices of inhibition occur much earlier before SSRT as previously thought (Raud and Huster, 2017). This is a challenge for many established signatures of inhibitory control, which occur close to the end of the stopping period indicated by traditional, potentially inflated SSRT estimates (Hanes et al., 1998; Wessel and Aron, 2015; Ogasawara et al., 2018). In this respect, it is notable that while the spatiotemporal coherence in fronto-central $\beta$-bursting after stop-signals took $\sim 200 \mathrm{~ms}$ to develop in the current study $(\sim 45 \mathrm{~ms}$ before the end of average SSRT), the FCz channel data show clear separation of successful and failed stop-trials even earlier than that (compare Fig. 2). In fact, the data show successful/failed differences as early as the time window that is centered $\sim 50 \mathrm{~ms}$ after the Stop-signal. This could reflect differences in the anticipatory recruitment of reactive stopping circuitry, which has been shown to partially underlie proactive control (Swann et al., 2012; Greenhouse and Wessel, 2013; van Belle et al., 2014), though this needs further testing in future studies.

The current study has key implications for future research. First, the precise neural origin of movement-related $\beta$-bursts needs to be investigated. Biophysical modeling has suggested that these bursts may results from the integration of nearsynchronous bursts of excitatory synaptic drive, targeting the dendrites of pyramidal neurons in specific cortical layers (Sherman et al., 2016). It remains to be tested whether the patches of cortex from which the scalp-recorded activity reported here are generated are subject to such layer-specific drive. Second, if $\beta$-bursting over sensorimotor sites is indeed indicative of a "tonic" inhibitory mode of the motor system, it would make it an 
interesting target for more systematic investigations of proactive inhibitory control that use different likelihoods of Stop-signals (Jaffard et al., 2008; Verbruggen and Logan, 2009; Greenhouse et al., 2012; Stuphorn and Emeric, 2012; Vink et al., 2015; Elchlepp et al., 2016). Third, there is great interest in pathological features of the $\beta$-frequency band in movement disorders, especially Parkinson's disease (Hammond et al., 2007; Bronte-Stewart et al., 2009; Jenkinson and Brown, 2011; Quinn et al., 2015). Recent studies have used trial-level $\beta$-burst measurements in subcortical areas (e.g., the subthalamic nucleus) to identify gait problems in PD (Anidi et al., 2018) and to develop adaptive brain stimulation approaches using closed-loop algorithms (Little et al., 2013; Tinkhauser et al., 2017). Future studies should aim to investigate whether similar pathological features can be found in scalprecorded $\beta$-bursts (Gauggel et al., 2004; van den Wildenberg et al., 2006; Obeso et al., 2011). Fourth, $\beta$-bursts may help elucidate the interactions between the subcortical aspects of the extrapyramidal motor system and the cortical areas governing higherorder motor control (Jenkinson and Brown, 2011; Leventhal et al., 2012; Bartolo and Merchant, 2015; Feingold et al., 2015). Indeed, it is tempting to hypothesize that $\beta$-bursting may be a "universal" language of the motor system, signifying distributed processing throughout the both pyramidal and extrapyramidal motor pathways.

Although the current study provides fundamentally novel insights into the neural underpinnings of movement in a large dataset, it also has shortcomings. First, because the neural activity in the current study was non-invasively recorded from the scalp, only limited conclusions can be drawn regarding the neural origins of the observed $\beta$-bursts. Second, although movement cancellation was clearly related to increased $\beta$-burst rates at fronto-central sites, $\beta$-bursts were neither necessary nor sufficient to cancel a movement on individual trials; at least when measured at the scalp-surface. Indeed, many failed Stop-trials also included $\beta$-bursts before SSRT. Moreover, not all successful Stop-trials included a suprathreshold $\beta$-burst before SSRT (Fig. $2 c$ ). Part of this outcome can likely be attributed to the imperfect signal-to-noise ratio of scalp-recordings. Indeed, intracranial recordings of the local field potential closer to its source will likely yield substantially higher $\beta$-burst rates. Furthermore, the search window for fronto-central $\beta$-bursts in this study was defined very conservatively. Although the time period between the Stop-signal and each participant's SSRT estimate is arguably the most theoretically motivated, straightforward window of interest, it is based on a variable, implicit measure (SSRT). Combined with the fact that frequency-resolved methods sacrifice some degree of temporal precision, behaviorally-relevant $\beta$-bursts may have fallen just outside of the detection window. Indeed, extending the window by a single $\beta$-cycle increased the number of detected fronto-central $\beta$-bursts on successful Stop-trials by $27 \%$, while the trial-differences remained highly significant (successful Stop vs Go: $t_{(233)}=12.07, p=2.27 \times 10^{-26}, d=0.94$; successful vs failed Stop: $\left.t_{(233)}=2.97, p=0.003, d=0.23\right)$. Future investigations, ideally using intracranial recordings, are necessary to investigate whether fronto-central $\beta$-bursts are truly necessary for stopping, and how the scalp-recorded $\beta$-burst signal relates to the original local field potential.

In summary, the current study found systematic changes in $\beta$-burst activity during both movement initiation and cancellation in humans. The data suggest that $\beta$-bursting over lateral sensorimotor sites reflects the inhibition of the underlying areas of the motor system, which has to be overcome during movement initiation, and which can be rapidly re-instated when movement cancellation is necessary (likely signaled by phasic increases in fronto-central $\beta$-bursts). This suggests that $\beta$-bursts are a fundamental signature of human motor control.

\section{References}

Anidi C, O’Day JJ, Anderson RW, Afzal MF, Syrkin-Nikolau J, Velisar A, Bronte-Stewart HM (2018) Neuromodulation targets pathological not physiological beta bursts during gait in Parkinson's disease. Neurobiol Dis 120:107-117.

Badry R, Mima T, Aso T, Nakatsuka M, Abe M, Fathi D, Foly N, Nagiub H, Nagamine T, Fukuyama H (2009) Suppression of human corticomotoneuronal excitability during the stop-signal task. Clin Neurophysiol 120:1717-1723.

Bartolo R, Merchant H (2015) beta oscillations are linked to the initiation of sensory-cued movement sequences and the internal guidance of regular tapping in the monkey. J Neurosci 35:4635-4640.

Bell AJ, Sejnowski TJ (1995) An information-maximization approach to blind separation and blind deconvolution. Neural Comput 7:1129-1159.

Benjamini Y, Krieger AM, Yekutieli D (2006) Adaptive linear step-up procedures that control the false discovery rate. Biometrika 93:491-507.

Boucher L, Palmeri TJ, Logan GD, Schall JD (2007) Inhibitory control in mind and brain: an interactive race model of countermanding saccades. Psychol Rev 114:376-397.

Brittain JS, Brown P (2014) Oscillations and the basal ganglia: motor control and beyond. Neuroimage 85:637-647.

Bronte-Stewart H, Barberini C, Koop MM, Hill BC, Henderson JM, Wingeier B (2009) The STN beta-band profile in Parkinson's disease is stationary and shows prolonged attenuation after deep brain stimulation. Exp Neurol 215:20-28.

Chikazoe J, Jimura K, Hirose S, Yamashita K, Miyashita Y, Konishi S (2009) Preparation to inhibit a response complements response inhibition during performance of a stop-signal task. J Neurosci 29:15870-15877.

Delorme A, Makeig S (2004) EEGLAB: an open source toolbox for analysis of single-trial EEG dynamics including independent component analysis. J Neurosci Methods 134:9-21.

Delorme A, Sejnowski T, Makeig S (2007) Enhanced detection of artifacts in EEG data using higher-order statistics and independent component analysis. Neuroimage 34:1443-1449.

Delorme A, Palmer J, Onton J, Oostenveld R, Makeig S (2012) Independent EEG sources are dipolar. PLoS One 7:e30135.

Doyle LM, Yarrow K, Brown P (2005) Lateralization of event-related beta desynchronization in the EEG during pre-cued reaction time tasks. Clin Neurophysiol 116:1879-1888.

Duque J, Greenhouse I, Labruna L, Ivry RB (2017) Physiological markers of motor inhibition during human behavior. Trends Neurosci 40:219-236.

Dutra IC, Waller DA, Wessel JR (2018) Perceptual surprise improves action stopping by non-selectively suppressing motor activity via a neural mechanism for motor inhibition. J Neurosci 38:1482-1492.

Dykstra T, Waller DA, Hazeltine E, Wessel JR (2019) Leveling the field for a fairer race between going and stopping: neural evidence for the race model of motor inhibition from a new version of the stop signal task. The MIT Press Journals, in press.

Elchlepp H, Lavric A, Chambers CD, Verbruggen F (2016) Proactive inhibitory control: a general biasing account. Cogn Psychol 86:27-61.

Engel AK, Fries P (2010) Beta-band oscillations: signalling the status quo? Curr Opin Neurobiol 20:156-165.

Feingold J, Gibson DJ, DePasquale B, Graybiel AM (2015) Bursts of beta oscillation differentiate postperformance activity in the striatum and motor cortex of monkeys performing movement tasks. Proc Natl Acad Sci U S A 112:13687-13692.

Fransen AM, van Ede F, Maris E (2015) Identifying neuronal oscillations using rhythmicity. Neuroimage 118:256-267.

Fransen AM, Dimitriadis G, van Ede F, Maris E (2016) Distinct alpha- and beta-band rhythms over rat somatosensory cortex with similar properties as in humans. J Neurophysiol 115:3030-3044.

Gauggel S, Rieger M, Feghoff TA (2004) Inhibition of ongoing responses in patients with Parkinson's disease. J Neurol Neurosurg Psychiatry 75:539544.

Greenhouse I, Wessel JR (2013) EEG signatures associated with stopping are sensitive to preparation. Psychophysiology 50:900-908.

Greenhouse I, Oldenkamp CL, Aron AR (2012) Stopping a response has 
global or nonglobal effects on the motor system depending on preparation. J Neurophysiol 107:384-392.

Hammond C, Bergman H, Brown P (2007) Pathological synchronization in Parkinson's disease: networks, models and treatments. Trends Neurosci 30:357-364.

Hanes DP, Patterson WF 2nd, Schall JD (1998) Role of frontal eye fields in countermanding saccades: visual, movement, and fixation activity. J Neurophysiol 79:817-834.

Jaffard M, Longcamp M, Velay JL, Anton JL, Roth M, Nazarian B, Boulinguez P (2008) Proactive inhibitory control of movement assessed by eventrelated fMRI. Neuroimage 42:1196-1206.

Jenkinson N, Brown P (2011) New insights into the relationship between dopamine, beta oscillations and motor function. Trends Neurosci 34:611-618.

Jha A, Nachev P, Barnes G, Husain M, Brown P, Litvak V (2015) The frontal control of stopping. Cereb Cortex 25:4392-4406.

Kaiser J, Birbaumer N, Lutzenberger W (2001) Event-related beta desynchronization indicates timing of response selection in a delayed-response paradigm in humans. Neurosci Lett 312:149-152.

Kühn AA, Williams D, Kupsch A, Limousin P, Hariz M, Schneider GH, Yarrow K, Brown P (2004) Event-related beta desynchronization in human subthalamic nucleus correlates with motor performance. Brain 127: 735-746.

Lee TW, Girolami M, Sejnowski TJ (1999) Independent component analysis using an extended infomax algorithm for mixed subgaussian and supergaussian sources. Neural Comput 11:417-441.

Leventhal DK, Gage GJ, Schmidt R, Pettibone JR, Case AC, Berke JD (2012) Basal ganglia beta oscillations accompany cue utilization. Neuron 73:523536.

Little S, Brown P (2014) The functional role of beta oscillations in Parkinson's disease. Parkinsonism Relat Disord 20:S44-S48.

Little S, Pogosyan A, Neal S, Zavala B, Zrinzo L, Hariz M, Foltynie T, Limousin P, Ashkan K, FitzGerald J, Green AL, Aziz TZ, Brown P (2013) Adaptive deep brain stimulation in advanced Parkinson disease. Ann Neurol 74:449-457.

Logan GD, Cowan WB (1984) On the ability to inhibit thought and action: a theory of an act of control. Psychol Rev 91:295-327.

Majid DS, Cai W, George JS, Verbruggen F, Aron AR (2012) Transcranial magnetic stimulation reveals dissociable mechanisms for global versus selective corticomotor suppression underlying the stopping of action. Cereb Cortex 22:363-371.

Matzke D, Love J, Wiecki TV, Brown SD, Logan GD, Wagenmakers EJ (2013) Release the BEESTS: Bayesian estimation of ex-Gaussian stopsignal reaction time distributions. Front Psychol 4:918.

McFarland DJ, Miner LA, Vaughan TM, Wolpaw JR (2000) Mu and beta rhythm topographies during motor imagery and actual movements. Brain Topogr 12:177-186.

Murthy VN, Fetz EE (1992) Coherent 25- to 35-Hz oscillations in the sensorimotor cortex of awake behaving monkeys. Proc Natl Acad Sci U S A 89:5670-5674.

Obeso I, Wilkinson L, Casabona E, Bringas ML, Álvarez M, Álvarez L, Pavón N, Rodríguez-Oroz MC, Macías R, Obeso JA, Jahanshahi M (2011) Deficits in inhibitory control and conflict resolution on cognitive and motor tasks in Parkinson's disease. Exp Brain Res 212:371-384.

Ogasawara T, Nejime M, Takada M, Matsumoto M (2018) Primate nigrostriatal dopamine system regulates saccadic response inhibition. Neuron 100:1513-1526.e4.

Oostenveld R, Fries P, Maris E, Schoffelen JM (2011) FieldTrip: open source software for advanced analysis of MEG, EEG, and invasive electrophysiological data. Comput Intell Neurosci 2011:156869.

Perrin F, Pernier J, Bertrand O, Echallier JF (1989) Spherical splines for scalp potential and current density mapping. Electroencephalogr Clin Neurophysiol 72:184-187.

Pfurtscheller G, Graimann B, Huggins JE, Levine SP, Schuh LA (2003) Spatiotemporal patterns of beta desynchronization and gamma synchronization in corticographic data during self-paced movement. Clin Neurophysiol 114:1226-1236.

Picazio S, Veniero D, Ponzo V, Caltagirone C, Gross J, Thut G, Koch G (2014) Prefrontal control over motor cortex cycles at beta frequency during movement inhibition. Curr Biol 24:2940-2945.

Quinn EJ, Blumenfeld Z, Velisar A, Koop MM, Shreve LA, Trager MH, Hill BC, Kilbane C, Henderson JM, Brontë-Stewart H (2015) Beta oscilla- tions in freely moving Parkinson's subjects are attenuated during deep brain stimulation. Mov Disord 30:1750-1758.

Raud L, Huster RJ (2017) The temporal dynamics of response inhibition and their modulation by cognitive control. Brain Topogr 30:486-501.

Ray NJ, Brittain JS, Holland P, Joundi RA, Stein JF, Aziz TZ, Jenkinson N (2012) The role of the subthalamic nucleus in response inhibition: evidence from local field potential recordings in the human subthalamic nucleus. Neuroimage 60:271-278.

Rossiter HE, Davis EM, Clark EV, Boudrias MH, Ward NS (2014) Beta oscillations reflect changes in motor cortex inhibition in healthy ageing. Neuroimage 91:360-365.

Sanes JN, Donoghue JP (1993) Oscillations in local field potentials of the primate motor cortex during voluntary movement. Proc Natl Acad Sci U S A 90:4470-4474.

Schall JD, Palmeri TJ, Logan GD (2017) Models of inhibitory control. Philos Trans R Soc Lond B Biol Sci 372:20160193.

Schmidt R, Leventhal DK, Mallet N, Chen F, Berke JD (2013) Canceling actions involves a race between basal ganglia pathways. Nat Neurosci 16:1118-1124.

Sherman MA, Lee S, Law R, Haegens S, Thorn CA, Hämäläinen MS, Moore CI, Jones SR (2016) Neural mechanisms of transient neocortical beta rhythms: converging evidence from humans, computational modeling, monkeys, and mice. Proc Natl Acad Sci U S A 113:E4885-E4894.

Shin H, Law R, Tsutsui S, Moore CI, Jones SR (2017) The rate of transient beta frequency events predicts behavior across tasks and species. eLife 6:e29086.

Stuphorn V, Emeric EE (2012) Proactive and reactive control by the medial frontal cortex. Front Neuroeng 5:9.

Swann N, Tandon N, Canolty R, Ellmore TM, McEvoy LK, Dreyer S, DiSano M, Aron AR (2009) Intracranial EEG reveals a time- and frequencyspecific role for the right inferior frontal gyrus and primary motor cortex in stopping initiated responses. J Neurosci 29:12675-12685.

Swann N, Poizner H, Houser M, Gould S, Greenhouse I, Cai W, Strunk J, George J, Aron AR (2011) Deep brain stimulation of the subthalamic nucleus alters the cortical profile of response inhibition in the beta frequency band: a scalp EEG study in Parkinson's disease. J Neurosci 31: 5721-5729.

Swann N, Cai W, Conner CR, Pieters TA, Claffey MP, George JS, Aron AR, Tandon N (2012) Roles for the pre-supplementary motor area and the right inferior frontal gyrus in stopping action: electrophysiological responses and functional and structural connectivity. Neuroimage 59: $2860-2870$.

Tenke CE, Kayser J (2005) Reference-free quantification of EEG spectra: combining current source density (CSD) and frequency principal components analysis (fPCA). Clin Neurophysiol 116:2826-2846.

Tinkhauser G, Pogosyan A, Little S, Beudel M, Herz DM, Tan H, Brown P (2017) The modulatory effect of adaptive deep brain stimulation on beta bursts in Parkinson's disease. Brain 140:1053-1067.

van Belle J, Vink M, Durston S, Zandbelt BB (2014) Common and unique neural networks for proactive and reactive response inhibition revealed by independent component analysis of functional MRI data. Neuroimage 103:65-74.

van den Wildenberg WP, van Boxtel GJ, van der Molen MW, Bosch DA, Speelman JD, Brunia CH (2006) Stimulation of the subthalamic region facilitates the selection and inhibition of motor responses in Parkinson's disease. J Cogn Neurosci 18:626-636.

van Ede F, Quinn AJ, Woolrich MW, Nobre AC (2018) Neural oscillations: sustained rhythms or transient burst-events? Trends Neurosci 41: $415-417$.

Verbruggen F, Aron AR, Band GP, Beste C, Bissett PG, Brockett AT, Brown JW, Chamberlain SR, Chambers CD, Colonius H, Colzato LS, Corneil BD, Coxon JP, Dupuis A, Eagle DM, Garavan H, Greenhouse I, Heathcote A, Huster RJ, Jahfari S, et al. (2019) A consensus guide to capturing the ability to inhibit actions and impulsive behaviors in the stop-signal task. eLife 8:e46323.

Verbruggen F, Logan GD (2008) Response inhibition in the stop-signal paradigm. Trends Cogn Sci 12:418-424.

Verbruggen F, Logan GD (2009) Proactive adjustments of response strategies in the stop-signal paradigm. J Exp Psychol Hum Percept Perform 35:835-854.

Vink M, Kaldewaij R, Zandbelt BB, Pas P, du Plessis S (2015) The role of 
stop-signal probability and expectation in proactive inhibition. Eur J Neurosci 41:1086-1094.

Wagner J, Wessel JR, Ghahremani A, Aron AR (2018) Establishing a right frontal beta signature for stopping action in scalp EEG: implications for testing inhibitory control in other task contexts. J Cogn Neurosci 30: 107-118.

Waller DA, Hazeltine E, Wessel JR (2019) Common neural processes during action-stopping and infrequent stimulus detection: the frontocentral P3 as an index of generic motor inhibition. Int J Psychophysiol. Advance online publication. Retrieved November 27, 2019. doi:10.1016/j.ijpsycho. 2019.01.004.

Wessel JR (2018a) A neural mechanism for surprise-related interruptions of visuospatial working memory. Cereb Cortex 28:199-212.

Wessel JR (2018b) Prepotent motor activity and inhibitory control de- mands in different variants of the go/no-go paradigm. Psychophysiology 55:e12871.

Wessel JR, Aron AR (2015) It's not too late: the onset of the frontocentral P3 indexes successful response inhibition in the stop-signal paradigm. Psychophysiology 52:472-480.

Wessel JR, Reynoso HS, Aron AR (2013) Saccade suppression exerts global effects on the motor system. J Neurophysiol 110:883-890.

Wessel JR, Ghahremani A, Udupa K, Saha U, Kalia SK, Hodaie M, Lozano AM, Aron AR, Chen R (2016) Stop-related subthalamic beta activity indexes global motor suppression in Parkinson's disease. Mov Disord 31:1846-1853.

Wessel JR, Waller DA, Greenlee JD (2019) Non-selective inhibition of inappropriate motor-tendencies during response-conflict by a frontosubthalamic mechanism. Elife 8:e42959. 